\title{
Editorial
}

\section{Hyperholomorphic Function Theory and Clifford Analyticity}

\author{
Kwang Ho Shon, ${ }^{1}$ Junesang Choi, ${ }^{2}$ and Tatsuhiro Honda ${ }^{3}$ \\ ${ }^{1}$ Department of Mathematics, Pusan National University, Busan 609-735, Republic of Korea \\ ${ }^{2}$ Department of Mathematics, Dongguk University, Gyeongju 780-714, Republic of Korea \\ ${ }^{3}$ Hiroshima Institute of Technology, Hiroshima 731-5193, Japan \\ Correspondence should be addressed to Kwang Ho Shon; khshon@pusan.ac.kr
}

Received 19 March 2014; Accepted 19 March 2014; Published 9 April 2014

Copyright (C) 2014 Kwang Ho Shon et al. This is an open access article distributed under the Creative Commons Attribution License, which permits unrestricted use, distribution, and reproduction in any medium, provided the original work is properly cited.

As a special issue of this highly esteemed journal, we were pleased to invite the interested authors to contribute their original research papers as well as good expository papers to this special issue that will make better improvement on the theory of Clifford analysis and its application to mathematical physics, providing new approaches to differential geometry using Clifford's geometric analysis. We suggested the following topics: theory of hyperholomorphic functions, regular functions, monogenic functions, hypercomplex number, dual number systems, spilt number systems, bicomplex numbers, and split biquaternions and pseudoquaternions; analytic extensions and applications; general theory of complex analytic spaces; complex partial differential operators, quaternion matrix equations, and generalized Cauchy-Riemann systems; domains of hyperholomorphy; and complex function spaces and hyperconjugate harmonic function. Certain papers that have significant results on complex analysis in the widest sense were intended to be welcome.

Besides some papers belonging to the above-intended topics, we are also happy to publish, in this special issue, several other papers regarding analytic number theory, $q$ series and combinatorics, and special functions and the theory of group representations.

\author{
Kwang Ho Shon \\ Junesang Choi \\ Tatsuhiro Honda
}




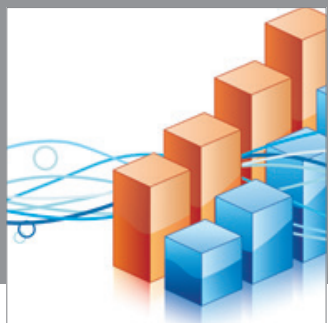

Advances in

Operations Research

mansans

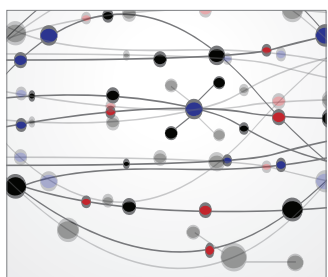

The Scientific World Journal
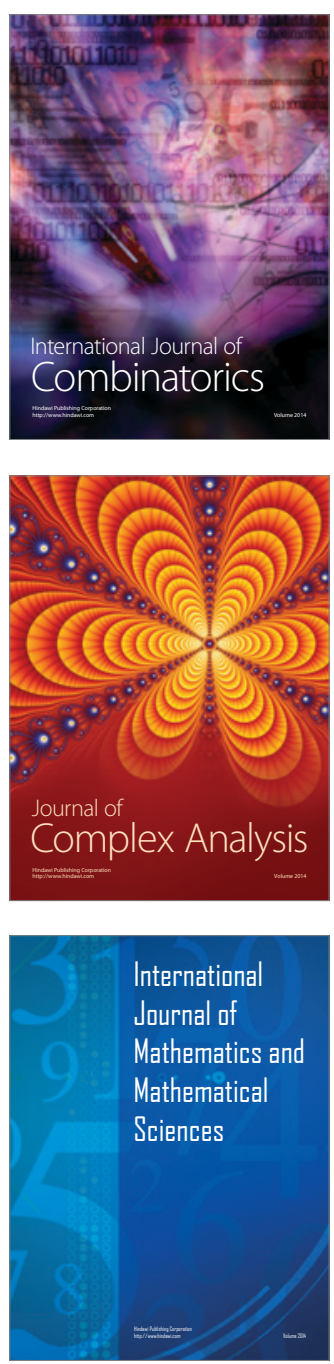
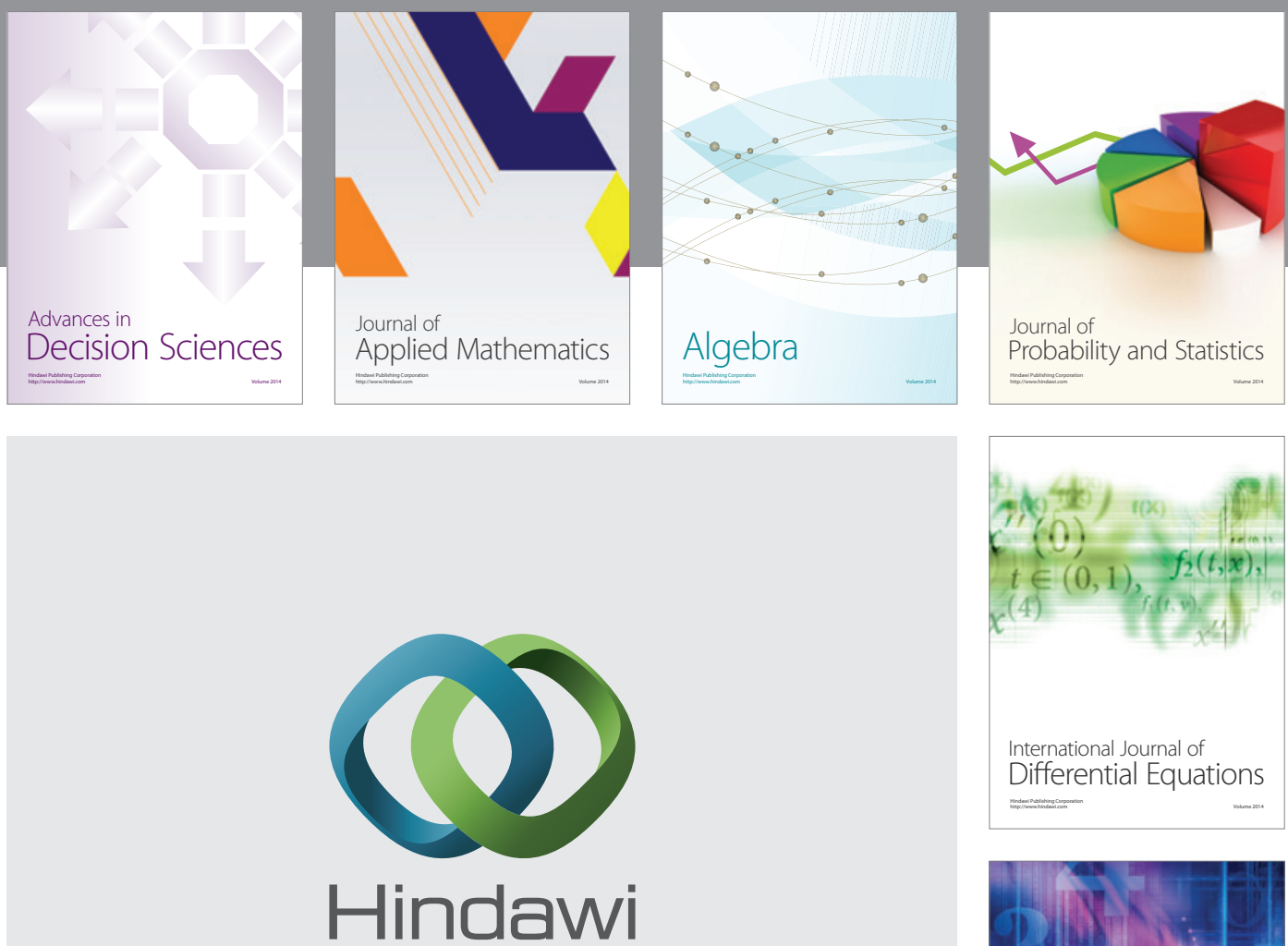

Submit your manuscripts at http://www.hindawi.com
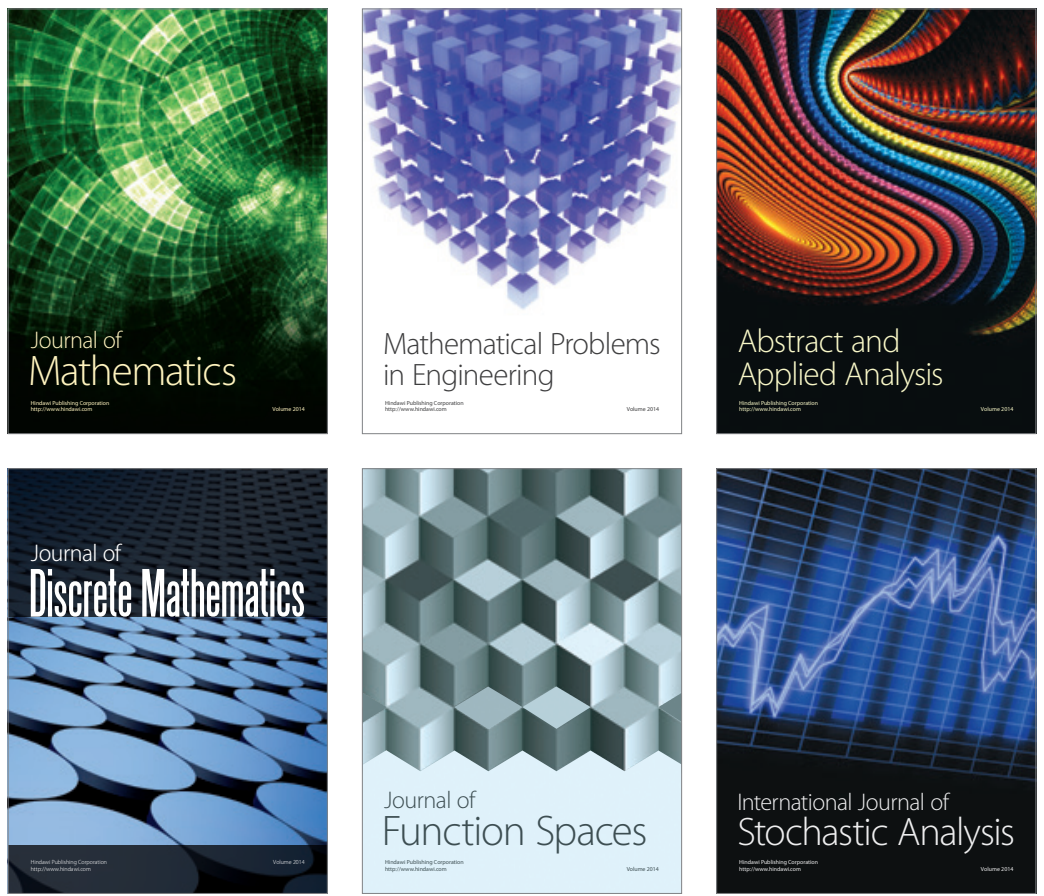

Journal of

Function Spaces

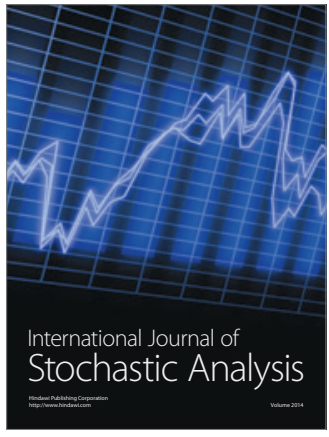

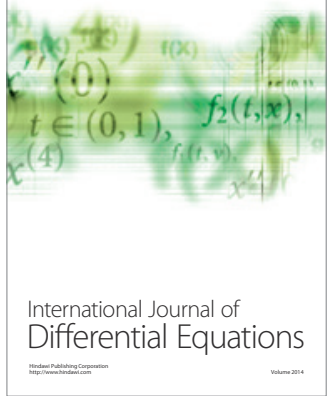
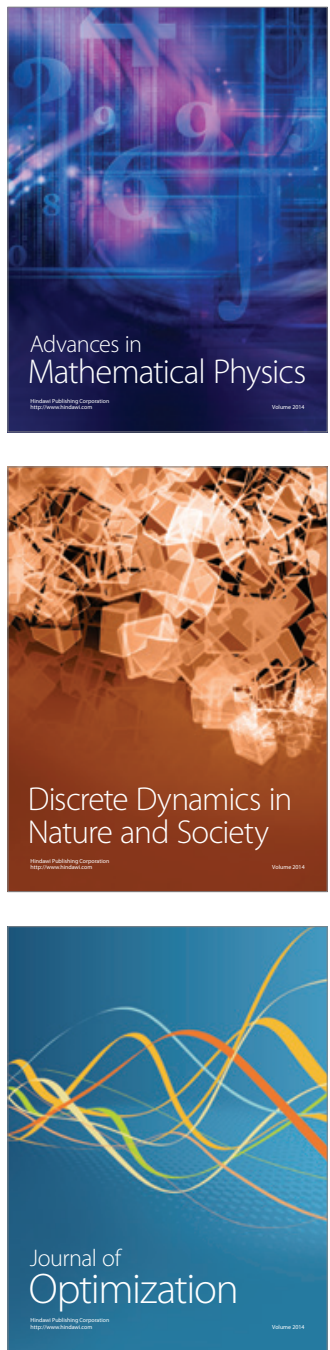